\title{
Zum 10-jährigen Bestehen der Deutschen Stiftung Friedensforschung - Friedensforschung für das 21. Jahrhundert
}

\author{
Michael Brzoska und Martina Fischer*
}

S eit ihrer Entstehung als eigenständige Forschungsrichtung in den 1950er Jahren ist die Friedensforschung in ihren Erkenntniszielen normativ ausgerichtet. Sie bewegt sich dabei zwischen zwei Extremen: der Suche nach Möglichkeiten der Verhütung von Krieg und der Sicherung globalen Überlebens. Niemand hat das so klar zum Ausdruck gebracht wie Johan Galtung, einer der Gründungsväter der Friedens- und Konfliktforschung. Er unterschied zwischen negativem und positivem Frieden und definierte ersteren als Abwesenheit von Krieg und letzteren als Überwindung von struktureller Gewalt zugunsten einer konstruktiven Gestaltung der Beziehungen zwischen Menschen und Gemeinwesen und ihrem Handeln im Einklang mit der Natur. ${ }^{1}$

Die Deutsche Stiftung Friedensforschung (DSF) hat diese Prinzipien teilweise aufgegriffen und sich für einen Mittelweg entschieden. In dem von der Stifterin im Jahr 2000 verfassten „Mission Statement“, das den Auftrag der DSF festlegt, heißt es zunächst, dass sie das „friedliche Zusammenleben der Menschen und Völker fördern" soll. ${ }^{2}$ Zudem wird ausgeführt, die Stiftung solle mithelfen, „Voraussetzungen und Bedingungen dafür zu schaffen, dass Krieg, Armut, Hunger, Unterdrückung verhütet, Menschenrechte gewahrt und die internationalen Beziehungen auf die Grundlage des Rechts gestellt werden“. Sie soll ferner darauf hinwirken, dass „die natürlichen Lebensgrundlagen und ihre Entwicklungsmöglichkeiten (...) auch für kommende Generationen erhalten werden".

Die DSF soll als Institution der Forschungsförderung also vorrangig zur Gewinnung von Erkenntnissen über die Verhinderung, Eindämmung und die Beendigung des gewaltsamen Austrags von zwischenstaatlichen und gesellschaftlichen Konflikten beitragen. Das schließt die Erforschung der vielfältigen gesellschaftlichen, ökonomischen, politischen und psychologischen Bedingungen für den friedlichen Austrag von Konflikten ebenso ein wie die Analyse von Ansätzen zur Eindämmung von Gewaltpotenzialen. Hier spielen Abkommen über Abrüstung und Rüstungskontrolle wie auch globale rechtliche

* Vorsitzender und Stellvertretende Vorsitzende der Deutschen Stiftung Friedensforschung. Eine frühere Fassung dieses Textes entstand für eine Broschüre zum 10-jährigen Bestehen der DSF.

1 Johan Galtung, A mini theory of peace, Lund 2007. http://www.transnational. org/ Resources_Treasures/2007/Galtung_MiniTheory.html.

2 Der Wortlaut entstammt dem „Stiftungsgeschäft“" zur Gründung der DSF vom 13. Oktober 2000.
Regelungen und politische Institutionen wie die Vereinten Nationen eine zentrale Rolle. Auch zehn Jahre nach Gründung der DSF ist dieser Auftrag weiterhin in hohem Maße aktuell.

Noch immer werden weltweit mehr als 30 Kriege geführt. ${ }^{3}$ Zwar belegt die Kriegsursachenforschung eine Abnahme von gewaltsam ausgetragenen Konflikten während der 1990er Jahre und bis in die zweite Hälfte der nachfolgenden Dekade. Seitdem ist dieser Trend jedoch wieder rückläufig. ${ }^{4}$ Besonders ernüchternd sind die zahlreichen Fehlschläge von Friedensabkommen und der erneute Rückfall von Nachkriegsgesellschaften in offene Feindseligkeiten. Diese Entwicklungen unterstreichen die Schwierigkeiten und komplexen Herausforderungen, die sich bei der Konsolidierung kriegszerstörter Gesellschaften ergeben. ${ }^{5}$ Außer in den von Medien und Politik viel diskutierten Konflikten in Afghanistan, Somalia und Kongo leiden Menschen noch in vielen anderen „vergessenen Kriegen“, etwa in Ostindien und im nördlichen Kaukasus. Zwar gibt es vereinzelt vielversprechende Bemühungen um Abrüstung und Rüstungskontrolle, aber diese vermögen gleichzeitig beobachtbare Trends zur Aufrüstung kaum einzudämmen.

Das globale Kriegsgeschehen gefährdet das Erreichen der Millenniums-Entwicklungsziele und die Verbesserung der Menschenrechtssituation in einer Reihe von Regionen. Vielfach werden ganze Gesellschaften in Mitleidenschaft gezogen und vor allem Zivilistinnen und Zivilisten zu Opfern. ${ }^{6}$

Weitere gewaltträchtige Konfliktdynamiken ergeben sich durch Veränderungen der globalen Machtarchitektur, durch den Zuwachs an wirtschaftlicher, weltpolitischer und militärischer

3 Die einschlägigen Kriegsstatistiken machen vor allem aufgrund unterschiedlicher Definitionen voneinander abweichende Angaben über die Anzahl der Konflikte, stimmen aber in der Bewertung der allgemeinen Trends überein. Für einen synoptischen Vergleich siehe Sven Chojnacki/Maurice Herchenbach/Gregor Reisch: Perspectives on War. Disentangling Distinct Phenomena: Wars and Military Interventions 1990-2008. In: Sicherheit und Frieden (S+F) 4/2009, S. 242-250.

4 Vgl. Tara Cooper/Sebastian Merz/Mila Shah: A more Violent World? Global Trends in Organized Violence, in: Beatrix Austin/Martina Fischer/Hans J. Gießmann (Hg.): Transforming Ethnopolitical Conflict. The Berghof Handbook Part II (forthcoming); der Beitrag reflektiert zentrale Erkenntnisse des Human Security Report 2009, ed. by Andrew Mack/Zoe Nielsen, Human Security Report Project, Vancouver 2010.

5 Vgl. Paul Collier et al.: Breaking the Conflict Trap: Civil War and Development Policy, New York: Oxford University Press and World Bank 2003, S. 83.

6 Über die Zahl ziviler Opfer wird heftig diskutiert, aber zweifellos übersteigt sie in jedem Jahr die der in Kämpfen getöteten Kombattanten um ein Vielfaches, siehe z. B. Adam Roberts: Lives and Statistics: Are 90\% of War Victims Civilians? In: Survival: Global Politics and Strategy 3/2010,S. 115-136. 
Bedeutung von Staaten wie China, Indien und Brasilien. Auch der zunehmende Wettbewerb um natürliche Ressourcen und Veränderungen der Umweltbedingungen sowie der Klimawandel wirken sich auf das Konfliktgeschehen aus.

Die Verschiebungen in den Konfliktdynamiken stellen die Friedensforschung vor immer neue Aufgaben, wobei „alte“ Themen deshalb keineswegs zwangsläufig als erledigt betrachtet werden können. Der Vorbeugung und Eindämmung kollektiver Gewalt und der Verbesserung der Bedingungen für einen friedlichen Konfliktaustrag kommt weiterhin eine zentrale Bedeutung zu. Außerdem ist es Aufgabe der Friedensforschung, neue Ideen und politische Instrumente, die für den Umgang mit Konflikten entwickelt werden, kritisch zu prüfen.

Neben der normativen Ausrichtung auf Gewaltminderung bildet die Praxisorientierung ein wesentliches Kennzeichen der Friedensforschung. Politik und Öffentlichkeit erwarten von ihr wissenschaftlich fundierte Antworten auf die Fragen, wie die Ursachen von Konflikten möglichst frühzeitig erkannt, wie Gewalteskalation verhindert und wie politische Regelungen für ihre Lösung gefunden werden können. Friedensforschung wendet sich in der Politikberatung gleichermaßen an Regierungen, Parlamente und politische Parteien wie auch an gesellschaftliche Organisationen und die breite Öffentlichkeit. Die nach anerkannten Standards des wissenschaftlichen Arbeitens gewonnenen Erkenntnisse müssen also jeweils in allgemein verständlicher Weise präsentiert werden, damit sie auch jenseits von Fachdiskursen Verbreitung finden.

Im Folgenden sollen zunächst die bisherigen Schwerpunktsetzungen und Orientierungen der Friedensforschung umrissen und auf ihre Zukunftstauglichkeit hin geprüft werden. Auf dieser Grundlage werden schließlich Themen erörtert, denen nach Einschätzung der Verfasser in den nächsten Jahren besondere Bedeutung zukommt.

\section{Wissenschaft Friedensforschung}

Friedensforschung ist ihrem Selbstverständnis nach eine moderne Wissenschaft. Sie bildet keine wissenschaftliche Disziplin im traditionellen Sinne mit eigenständigen Forschungsmethoden. Wie andere neue Zweige der wissenschaftlichen Forschung, etwa die Umweltforschung oder die Neurowissenschaft, hat sie sich durch die Integration unterschiedlicher Forschungstraditionen zu einem Kernthema von hohem gesellschaftlichem Rang entwickelt. Friedensforschung ist von der Anlage her transdisziplinär. Sie zielt darauf ab, die relevanten Erkenntnisse und Methoden zur Bearbeitung des zentralen Themas der Gewaltminderung in einer Weise zusammenzuführen, die über die traditionellen wissenschaftlichen Einzeldisziplinen hinausweist und einen Mehrwert erbringt.

Um diesem hohen Anspruch gerecht werden zu können, ist die Friedensforschung auf den ständigen Austausch mit unterschiedlichen Wissenschaftsbereichen und den Dialog mit vielen Disziplinen angewiesen. Deren Erkenntnisse und Methoden müssen möglichst rasch Eingang in die Friedensforschung finden. Ebenso sollten sich Ergebnisse der Friedensforschung der kritischen Überprüfung in anderen Wissenschaften stellen. Auch ihr Selbstverständnis muss sich kritisch hinterfragen lassen. ${ }^{7}$

Tatsächlich können viele unterschiedliche Disziplinen Erkenntnisse zur Gewaltminderung und zu den Voraussetzungen für einen gewaltfreien Konfliktaustrag liefern. Derzeit ist die Politikwissenschaft der wichtigste akademische Partner der institutionellen Friedensforschung. Das war nicht immer so. In ihrer Entstehungsphase Anfang der 1960er Jahre dominierte die Soziologie und zahlreiche einflussreiche Beiträge kamen darüber hinaus aus der Geschichtswissenschaft, Psychologie, Pädagogik, Physik und Rechtswissenschaft. ${ }^{8}$ Hier schlummert möglicherweise noch erhebliches Potenzial. So hat die Soziologie beispielsweise in der Forschung zu Fragen der internationalen Beziehungen und transnationalen Sicherheit in den letzten Jahren einen deutlichen Aufschwung erlebt. ${ }^{9}$ Ähnliches gilt für die Neurowissenschaft. Ihre Erkenntnisse haben z.B. in der Ökonomie zu lebhaften Diskussionen beigetragen; sie sind in der Friedensforschung jedoch bisher nicht sichtbar geworden.

Die DSF hat sich seit ihrem Bestehen erfolgreich darum bemüht, den interdisziplinären Austausch zu unterstützen. Sie hat durch die Förderung von Forschungsprojekten, wissenschaftlichen Tagungen und Vernetzungsaktivitäten maßgeblich hierzu beigetragen und darüber hinaus im Rahmen der Strukturund Nachwuchsförderung wichtige Impulse dafür geliefert. So wurde zum einen der Aufbau interdisziplinärer Studiengänge unterstützt, zum anderen wurden durch die Förderung zweier Stiftungsprofessuren in Hamburg und Darmstadt gezielt Brücken zwischen der hierzulande bislang sozialwissenschaftlich geprägten Friedensforschung und den Naturwissenschaften geschlagen.

In der von der DSF angestoßenen Forschungsinitiative zur Rolle von Religion in der Dynamik von Kriegen und Bürgerkriegen und in Friedensprozessen erwies sich wiederum der Austausch mit Religions- und Kulturwissenschaftlern als gewinnbringend. So konnten eindimensionale Annahmen über Konflikt-

\footnotetext{
7 Siehe zum Beispiel die von der Arbeitsgemeinschaft für Friedens- und Konfliktforschung unterstützte Bestandsaufnahme der Wissensbestände der Friedensforschung: Peter Imbusch/Peter Schlotter/Simone Wisotzki: Friedens- und Konfliktforschung, Baden-Baden: Nomos 2010. Eine Fülle von Perspektiven bieten Astrid Sahm/Manfred Sapper/Volker Weichsel (Hg.): Die Zukunft des Friedens. Eine Bilanz der Friedens- und Konfliktforschung. 2. Auflage, Wiesbaden: VS Verlag, 2007. Für kritische Reflexionen siehe Matti Jutila/Samu Pehkonen/Tarja Väyrynen: Resuscitating a Discipline: An Agenda for Critical Peace Research Millennium, in: Journal of International Studies 3/2008, S. 623-640; Lothar Brock: Die Arbeit am Frieden als Verstrickung in den Krieg. Von der kritischen zur pragmatischen Friedensforschung?, in: Marcel Baumann et al. (Hg.): Friedensforschung und Friedenspraxis. Ermutigung zur Arbeit an der Utopie. Festschrift für Reiner Steinweg zum 70. Geburtstag. Frankfurt am Main: Brandes \& Apsel 2009, S. 27-41.

8 Gemessen an den Hinweisen auf die Autorinnen und Autoren von Beiträgen in den Zeitschriften Journal of Peace Research, Journal of Conflict Resolution, Peace and Change, Conflict Management and Peace Science, Friedenswarte, Friedensanalysen, Sicherheit und Frieden sowie Wissenschaft und Frieden.

9 Ein Ausdruck davon ist die Neugründung der International Political Sociology, einer neuen Zeitschrift der International Studies Association.
} 
ursachen, die z.B. mit der These vom „Zusammenprall der Kulturen “10 populär wurden, empirisch widerlegt werden. ${ }^{11}$

Weitere Ansätze der interdisziplinären Verknüpfung wären wünschenswert. So sollten Themen der Gewaltprävention vor allem in der Wirtschaftswissenschaft in Deutschland stärker reflektiert werden. Im angloamerikanischen Raum und in Skandinavien hat die wirtschaftswissenschaftliche Forschung zu Bürgerkriegen stark zugenommen. Dies wurde in Deutschland vor allem in der Politikwissenschaft und Friedensforschung aufgenommen, fand aber in der Wirtschaftswissenschaft bislang kaum Beachtung. ${ }^{12}$

Da die begrenzten finanziellen Mittel der DSF in absehbarer Zeit keine weiteren Maßnahmen der Struktur- und Nachwuchsförderung zulassen, muss sich die DSF in Zukunft zunächst darauf konzentrieren, den Austausch zwischen unterschiedlichen Disziplinen durch Anreize im Rahmen der Projektförderung zu unterstützen. Interdisziplinarität sollte weiterhin ein wichtiges Kriterium der Förderpolitik bilden, zumal interdisziplinäre Forschungsvorhaben bei anderen Fördereinrichtungen nur schwer Akzeptanz finden. Die Bevorzugung trans- und interdisziplinärer Anträge könnte die DSF in der Förderlandschaft herausheben und zu ihrer Profilbildung beitragen. Dies würde allerdings eine Erhöhung ihrer Finanzausstattung erforderlich machen, die es zulässt, auch Forschungsprojekte mit mehreren Projektstellen unter Einbeziehung von Experten aus unterschiedlichen Disziplinen zu finanzieren.

\section{2. "Alte“ Themen mit neuen Perspektiven}

\section{Gewaltprävention}

Die Zahl zwischenstaatlicher Kriege ist seit den 1970er Jahren langsam aber kontinuierlich zurückgegangen. Das bedeutet jedoch nicht, dass solche Kriege nicht mehr stattfinden. Wie das Beispiel Äthiopien und Eritrea 1999-2000 zeigte, fordern sie teilweise sogar sehr viele Opfer. Die dominante Form der kollektiven bewaffneten Gewalt bilden allerdings inzwischen innerstaatliche Kriege. Die Zahl der „Bürgerkriege“ nahm nach dem Ende des 2. Weltkrieges bis Mitte der 1990er Jahre kontinuierlich zu, bevor sie dann über ein Jahrzehnt hin deutlich sank. Die Forschung ist sich nicht darüber einig, welche Faktoren dazu geführt haben, dass zunächst internationale Kriege und dann auch innerstaatliche Kriege seltener wurden. Es wird unter anderem auf die Bedeutung internationaler Organisationen, die zunehmende Verbreitung von Demokratie, die wachsende internationale Verflechtung in Wirtschaft und Kommunikation und auf das global zunehmende Bewusstsein des Zusam-

10 Vgl. Samuel P. Huntington: The Clash of Civilizations and the Remaking of World Order. New York: Simon \& Schuster, 1998.

11 Vgl. Volker Rittberger, Auf dem Weg zum nachhaltigen Frieden? Aufgaben der Friedensforschung zu Beginn des 21. Jahrhunderts, in Sicherheit und Frieden 1, (27), 2009, . S. 47-54 (hier S. 50). Vgl. auch Matthias Basedau/Peter Körner: Zur ambivalenten Rolle von Religion in afrikanischen Gewaltkonflikten. Osnabrück 2009 (Forschung DSF Nr. 18); Andreas Hasenclever/Alexander de Juan: Religionen in Konflikten. Eine Herausforderung für die Friedenspolitik. In: Aus Politik und Zeitgeschichte. 6/2007, S. 10-16.

12 Siehe z.B. den Überblick in Christopher Blattman/Edward Miguel: Civil War, in: Journal of Economic Literature 1/2010, S. 3-57. menlebens in einer Welt als Einflussfaktoren hingewiesen. ${ }^{13}$ Zahlreiche in den letzten Jahren erarbeitete internationale Studien kommen in dieser Frage zu durchaus unterschiedlichen Ergebnissen. ${ }^{14}$

Allerdings scheint es verfrüht, so wie der US-amerikanische Politikwissenschaftler John Mueller ${ }^{15}$ von der „Obsoleszenz“ des Krieges zu sprechen. Schließlich deuten neuere Daten darauf hin, dass sich der Trend seit einigen Jahren wieder umkehrt und die Zahl der innerstaatlichen Kriege wieder steigt. ${ }^{16}$ Auch die Zuspitzungen von Konfliktsituationen in einer Reihe von Regionen - um nur einige wenige aktuelle Beispiele zu nennen: koreanische Halbinsel, Sudan, Somalia, Israel/Palästina, Iran - sprechen gegen einen übertriebenen Optimismus. Die Untersuchung dieser Konfliktherde und die Suche nach Möglichkeiten friedlicher Konfliktbeilegung bleiben somit von zentraler Bedeutung für die Friedensforschung und damit auch für die DSF.

Parallel zur Diskussion über die Trends im Kriegsgeschehen sind aber auch neue Fragen aufgetaucht, deren Bearbeitung noch nicht sehr weit gediehen ist. ${ }^{17}$ Dazu gehört die Frage, ob es eine Art „Substitutionseffekt“ der Gewalt gibt. Es wird vermutet, dass möglicherweise erfolgreiche Beispiele der Kriegsverhütung und -beendigung mit einer Zunahme von niedrigschwelliger Gewalt einhergehen. Diese These ist etwa im Zusammenhang mit der Ausweitung terroristischer Anschläge, insbesondere in Regionen wie dem Nahen Osten, vorgetragen worden, wo militante Gruppen militärisch übermächtigen Gegnern gegenüberstehen. Eine andere Frage ist, ob sich Erkenntnisse der Forschung zu den Ursachen von innerstaatlichen Kriegen auch auf niedrigschwellige Gewaltformen, beispielsweise „Brotaufstände“, übertragen lassen, oder ob diese mit anderen Faktoren wie etwa der ungleichen Verteilung von Reichtum und Einkommen oder wirtschaftlichen Aspekten der Globalisierung besser erklärt werden können. Auch die Frage der Bedeutung von Gier („greed“) oder politischen bzw. sozialen Benachteiligungen („grievances“) für die Entstehung von Gewaltkonflikten und die Gewichtung dieser Faktoren wurden in der Friedensforschung kontrovers diskutiert, ohne dass es dazu abschließende konsensuale Ergebnisse gibt. ${ }^{18}$

13 Andrew Mack weist darauf hin, dass mit dem Ende des Kolonialismus und des Ost-West-Konflikts zentrale Konfliktursachen entfielen. Zudem führt er die positive Entwicklung auf die aktive Politik der Friedensschaffung und Friedenskonsolidierung zurück, die während der 1990er Jahre von den Vereinten Nationen und weiteren Regionalorganisationen, Regierungen und Nichtregierungsorganisationen umgesetzt wurde. Vgl. Andrew Mack: Global Political Violence. Explaining the Post-Cold War Decline, in: Volker Rittberger und Martina Fischer (eds.), Strategies for Peace, Contributions of International Organisations, States, and Non-State Actors, Opladen und Farmington Hills, 2008, 75-107 (hier S. 79).

14 Vgl. für eine kritische Bestandsaufnahme: Anna Geis/Harald Müller/Wolfgang Wagner (Hg.): Schattenseiten des demokratischen Friedens. Frankfurt a. M.: Campus 2007.

15 John Mueller: Retreat from Doomsday: The Obsolescence of Major War. New York: Basic Books 1989, und ders.: The Remnants of War. Ithaca: Cornell University Press 2007.

16 Vgl. Tara Cooper et al., (forthcoming), a.a.O.

17 Einen Überblick über den Wandel im Verständnis von Krieg gibt Anna Geis (Hg.): Den Krieg überdenken. Kriegsbegriffe und Kriegstheorien in der Kontroverse. Baden-Baden: Nomos 2006

18 Vgl. Paul Collier/Anke Hoeffler: Greed and Grievance in Civil War. The World Bank Policy Research Working Paper 2355, May 2000; Mats Berdal/David M. Malone (Hg.): Greed and Grievance: Economic Agendas in Civil Wars. Boulder (Colorado): Lynne Rienner Publishers 2000; Anthony Vinci: Greed-Grievance Reconsidered: The Role of Power and Survival in the Motivation of Armed Groups, in: Civil Wars 1/2006 S. 25-45. 
Insbesondere mit Bezug auf Lateinamerika, aber zunehmend auch auf andere Regionen wird danach gefragt, ob und wie kriminelle und politisch motivierte kollektive Gewalt miteinander verknüpft sind. Besondere Aufmerksamkeit hat in den letzten Jahren das Phänomen der „Privatisierung“ von Gewalt erfahren, also die zunehmende Bedeutung privater Militärund Sicherheitsfirmen in Kriegsgebieten. Nachdem zunächst vor allem rechtliche Aspekte untersucht wurden, nimmt inzwischen die Zahl empirischer Untersuchungen zu, die sich auch den organisationssoziologischen und ökonomischen Aspekten dieses Problems widmen. Dabei zeigt sich die Komplexität der Arrangements, mit denen Gewalt eingehegt, aber auch erzeugt wird. ${ }^{19}$ Ein weiteres Forschungsdesiderat betrifft die Frage, wann und warum Massaker an Zivilisten bis hin zu Völkermorden und anderen einseitigen Gewalttaten in Kriegen verübt werden. Ein Grundproblem aller Forschungsanstrengungen zu „alternativen“ Gewaltformen - alternativ zu Kriegen - ist der Mangel an klaren Konzepten zur Unterscheidung und Einordnung der verschiedenen Gewaltphänomene. Eine andere Schwierigkeit resultiert aus dem Mangel an belastbaren Informationen und Daten. ${ }^{20}$ Daraus ergibt sich ein wichtiges Forschungsdesiderat, zumal derartige Erkenntnisse für die politische Praxis, vor allem in der Entwicklungszusammenarbeit, von großer Bedeutung sind. ${ }^{21}$

\section{Abrüstung, Rüstungskontrolle und neue Rüstungstechnologien}

Mit der partiellen Wiederbelebung internationaler Abrüstung und Rüstungskontrolle - aktuelle Beispiele sind das Oslo-Abkommen zu Streumunition, das „New Start“-Abkommen zwischen den USA und Russland und die wieder auflebenden Bemühungen um konventionelle Rüstungskontrolle in Europa stellen sich „alte“ Fragen von Rüstungskontrolle und Abrüstung neu.

Das betrifft zunächst das Verhältnis nuklearer zu konventioneller Rüstung. Mit dem zahlenmäßigen Abbau von Nuklearwaffen gewinnen konventionelle Waffen möglicherweise in der Wahrnehmung von militärischen Befehlshabern und politischen Entscheidungsträgern wieder an Bedeutung. In Russland wird schon jetzt die Befürchtung geäußert, dass bei weiterer nuklearer Abrüstung die USA und China militärisch an Schlagkraft gewinnen, während Russland verlieren wird. In den USA wird die Ansicht vertreten, dass Staaten, die derzeit durch entsprechende Zusicherungen unter dem amerikanischen „Atomschirm“ stehen, im Zuge der Abschaffung von Nuklearwaffen konventionell aufrüsten würden.

19 Exemplarisch dafür können die auch von der DSF geförderten Untersuchungen von Andreas Mehler stehen, der den problematischen aber innovativen Begriff des Gewaltoligopols geprägt hat. Siehe etwa Andreas Mehler: "Gewaltoligopole“ und Sicherheit als „kollektives Gut“ - Konzeptionelle Überlegungen (mit Illustrationen aus Westafrika), in: Matthias Basedau/Hanspeter Mattes/Anika Oettler (Hg.): Multiple Unsicherheit. Befunde aus Asien, Nahost, Afrika und Lateinamerika, Hamburg: Deutsches Überseeinstitut 2005, S. 193-208

20 Siehe dazu etwa: Michael Brzoska: Collective Violence beyond the Standard Definition of Armed Conflict. In: Stockholm International Peace Research Institute, SIPRI Yearbook 2007, Oxford: Oxford University Press 2007, S. 94104.

21 Siehe die Übersicht in: Geneva Declaration: The Global Burden of Armed Violence Reduction. Small Arms Survey, Genf 2008.
Hier wird erneut eine Frage aufgeworfen, welche die Friedensforschung schon in der Vergangenheit intensiv beschäftigt hat und stets zu heftigen Kontroversen Anlass gab: Kann nukleare Abschreckung Krieg verhindern oder ist dies nur eine Illusion? Ist die Vermeidung eines offenen Krieges in Europa während des „Kalten Krieges“ auf die Logik der Abschreckung und die Verfügungsgewalt einzelner Mächte über Atomwaffen zurückzuführen, oder ist es eher ein historischer Glücksfall, dass diese Waffen nicht zum Einsatz gebracht wurden? ${ }^{22}$

Diese Frage wird sich vielleicht nie abschließend mit wissenschaftlichen Methoden klären lassen. Gleichwohl haben die zentralen politischen Akteure, die über die Vorhaltung oder den Einsatz von Massenvernichtungswaffen entscheiden, unterschiedliche Interpretationen hierzu. Diese Erklärungsmuster und die Beschreibung aktueller militärischer Kräfteverhältnisse dürften für die Frage, inwieweit die nukleare und konventionelle Abrüstung und Rüstungskontrolle weiterhin Fortschritte machen können, von großer Bedeutung sein. Daher ist es wichtig zu untersuchen, von welchen Interessenlagen und Wahrnehmungen Entscheidungsträger in Verhandlungsprozessen geleitet werden und wie diese Muster verändert bzw. beeinflusst werden können.

Wissenschaft, die sich der Gewaltprävention und -minderung widmet, ist gefordert, über neue und alternative Ansätze der Rüstungskontrolle und ihre Erfolgsbedingungen zu reflektieren. Seit den 1990er Jahren hat die „humanitäre Rüstungskontrolle“ eine rasante Entwicklung genommen. Immerhin führten internationale Kampagnen zu Einschränkungen des Einsatzes von Minen und Brandwaffen, zum Verbot von Antipersonenminen, Blendlasern und vielen Typen von Streumunition. Es wäre zu prüfen, ob der humanitäre Ansatz auf weitere Waffentypen ausdehnbar ist. Außerdem muss der Frage nachgegangen werden, ob völkerrechtliche Normen und Verbote wirklich Gewaltminderung befördern und inwiefern sie die großen Militärmächte überhaupt binden können. Auffällig ist die große Bedeutung von Nichtregierungsorganisationen (NRO) in der humanitären Rüstungskontrolle. Es fragt sich, ob diese auch in anderen Feldern der Rüstungskontrolle und Abrüstung vergleichbaren Einfluss gewinnen und ob sie auch Funktionen in der Verifikation von Abkommen übernehmen können.

Manche Erfolge in der Rüstungskontrolle und Abrüstung wurden nachträglich durch neue Waffensysteme wieder zunichte gemacht. Die DSF hat im Bereich der Strukturbiologie, der Nanotechnologie und der Robotik bereits Ansätze zur kritischen Analyse solcher technologischer Trends gefördert. ${ }^{23}$ Weitere friedensgefährdende Technologien werden mit großer Wahrscheinlichkeit entwickelt werden. Diese Innovationen und die

22 Siehe etwa Dieter Senghaas: Abschreckung und Frieden. Studien zur Kritik organisierter Friedlosigkeit, Frankfurt am Main: Europäische Verlagsanstalt 1969 und Wolf Graf von Baudissin: Abschreckung durch Kernwaffen?, in: Manfred Lahnstein/Hans Matthöfer: Leidenschaft zur praktischen Vernunft. Helmut Schmidt zum 70. Berlin: Siedler-Verlag 1989, S. 35-50.

23 Vgl. hierzu Alexander Kelle/Kathryn Nixdorff/Malcolm Dando: A Paradigm Shift in the CBW Proliferation Problem: Devising Effective Restraint on the Evolving Biochemical Threat. Osnabrück 2008 (Forschung DSF Nr. 12); Jürgen Altmann, Nanotechnology and Preventive Arms Control, Forschung DSF No. 3, Osnabrück: Deutsche Stiftung Friedensforschung, 2005 und Jürgen Altmann: Millimetre Waves, Lasers, Acoustics for Non-Lethal Weapons? Physics Analyses and Inferences. Osnabrück 2008 (Forschung DSF Nr. 16). 
von ihnen ausgehenden Kriegsgefahren müssen auch in $\mathrm{Zu}$ kunft Gegenstand kritischer Friedensforschung sein.

\section{Friedenskonsolidierung}

Seit den frühen 1990er Jahren wurden zahlreiche internationale Bemühungen der Friedenskonsolidierung in Nachkriegsregionen unternommen. Diese richteten sich meist auf die Unterstützung demokratischer Wahlen, den Aufbau von Verwaltungen und die Reform von Polizei und Militär, die Einrichtung von Menschenrechtskommissionen, Antikorruptionskampagnen, den Aufbau alternativer Medien sowie bildungs- und kulturpolitische Maßnahmen zur Unterstützung der Aussöhnung verfeindeter Gemeinwesen. Teilweise wurde auch versucht, Ansätze der Konfliktprävention mit Entwicklungspolitik zu verknüpfen. Einschlägige Organisationen wie etwa der Entwicklungsausschuss der OECD haben versucht, daraus handbuchartige Modelle für „best practice“ zu entwickeln. Beispiele wie Afghanistan oder Kongo zeigen jedoch, dass viele der Bemühungen zweifelhafte Ergebnisse zeitigen. In der Forschung zu Gewaltkonflikten ist deutlich geworden, dass Defizite in der Friedenskonsolidierung ein wesentlicher Bedingungsfaktor für erneute Gewalt sind. Einige Wissenschaftler sprechen von der „Konfliktfalle“, in der einzelne Regionen ohne erfolgreiche Friedensprozesse gefangen bleiben.

Daher wurden in den letzten Jahren gängige Modelle des „nation building " und damit verbundene Ansätze der Friedenskonsolidierung, die als „liberal peace“ bezeichnet werden, heftig kritisiert. ${ }^{24}$ Die Kritik richtete sich unter anderem auf die Übertragung von Modellen der Staatlichkeit ${ }^{25}$ und Demokratisierung, die sich an westlichen Industrieländern orientieren, aber für viele Gesellschaften außerhalb der OECD-Welt als unangemessen empfunden werden, zumal kulturelle Bezüge und gewachsene Institutionen in der Regel ignoriert und gar nicht erst auf ihre Bedeutung für Prozesse der Gemeinwesenentwicklung und Aussöhnung hin geprüft werden. Vor allem Interventionen, die frühzeitige Wahlen an den Anfang von Friedenskonsolidierung stellen, noch bevor politische und rechtliche Institutionen ansatzweise aufgebaut werden konnten, wurden massiv in Frage gestellt. ${ }^{26}$ Zahlreiche Beispiele, angefangen von Bosnien-Herzegowina bis hin zu Afghanistan, haben gezeigt, dass sich ein solches Vorgehen kontraproduktiv auf den Friedensaufbau und teilweise konfliktverlängernd auswirken kann. Kritisch reflektiert wurde auch das in sich widersprüchliche Verhalten der externen Akteure, die zum Beispiel Demokratisierung propagieren, aber selbst ihre Entscheidungen in den betroffenen Regionen losgelöst von demokratischer Kontrolle träfen oder sich internationaler Rechtsprechung entzögen.

24 Siehe etwa Roland Paris: At War's End. Building Peace after violent Conflict. Cambridge: Cambridge University Press, 2004; Roland Paris/Donald T. Sisk: The Dilemmas of Statebuilding. Confronting the Contradictions of Postwar Peace Operations. London: Routledge 2009; Oliver Richmond: Reconstructing the Liberal Peace, in: Volker Rittberger/Martina Fischer 2008, a.a.O., S. 49-74.

25 Eine kritische Einschätzung der Debatte über Staatszerfall, „fehlgeschlagene Staaten" und „nation building“ aus historischer Sicht liefert Ekkehard Krippendorff: Staat muss sein. Muss Staat sein?, in: Aus Politik und Zeitgeschichte pendorff: Staat mus

26 Vgl. Roland Paris 2004, a.a.O.
Eine weitere Kritiklinie richtet sich auf den von den externen Mächten ausgeübten Druck zur Anpassung von Gesellschaften an die Erfordernisse internationaler Finanz- und Geberinstitutionen. Die einfache Gleichung, dass die Etablierung von Regierungen und Parlamenten bei gleichzeitiger wirtschaftlicher Liberalisierung (oder auch überstürzter Privatisierung von öffentlichem Eigentum) automatisch Frieden und Stabilität erzeuge, geht offensichtlich vielerorts nicht auf. Der Zwang zur Anpassung an die Realitäten der im Modell propagierten wirtschaftlichen Globalisierung erschwert überdies jede nachholende wirtschaftliche Entwicklung.

Die internationale Politik steht aktuell vor der Herausforderung, dem weiteren Zerfall von fragilen Staaten durch aufeinander abgestimmte Maßnahmen entgegenzuwirken und zum anderen den Wieder- oder Neuaufbau staatlicher Strukturen in besser angepasster Weise zu fördern. ${ }^{27}$ Gleichzeitig steht sie vor dem Problem, dass staatliche Strukturen, wie sie etwa in den OECD-Staaten gegeben sind, in weiten Teilen der Welt nie oder nur fragmentiert in einzelnen Regionen und Sektoren existierten und auch in der Zukunft kaum realisiert werden können. Oft sind externe Friedensbemühungen mit hybriden Ordnungen konfrontiert, in denen sich moderne und vormoderne gesellschaftliche Organisationsprinzipen überlagern. ${ }^{28}$ Diese lassen sich nicht ohne Weiteres mit Begriffen wie „Staatlichkeit“ oder „Anarchie“ erfassen. In den vergangenen Jahren wurde verstärkt über die Frage des Umgangs mit solchen hybriden Ordnungen nachgedacht; Fallstudien zu Friedensprozessen aus zahlreichen Weltregionen vom Pazifik bis zum Balkan wurden vor dem Hintergrund des „Liberal peace“-Paradigmas diskutiert. Dabei wurde deutlich, dass neben zahlreichen Beispielen gescheiterter Interventionen (etwa in Afghanistan) und zweifelhafter Effekte (eingefrorene Konflikte wie z.B. in Bosnien) in einigen Fällen aber auch Lernprozesse und erfolgreiche Ansätze der Friedenskonsolidierung (z.B. in der Pazifikregion auf Bougainville/Papua Neuguinea und den Salomonen) erkennbar sind. ${ }^{29}$

Die Kritik am „liberal peace“ hat grundlegende Defizite der Friedenskonsolidierung aufgedeckt, ohne ein umsetzbares Gegenmodell zu entwickeln. Das ist das Recht von Kritikern - allerdings bleibt dies angesichts der Notwendigkeit erfolgreicher Friedenskonsolidierung unbefriedigend. Solange keine grundlegend neue Theorie der Transformation von einer Kriegs- zu einer Friedensgesellschaft in Sicht ist, müssen weiter Teilaspekte untersucht werden - auf der Grundlage einer kritischen Sicht auf gängige liberale Theorien.

Weiterhin stellen sich die Fragen: Welche Ansätze der Friedensförderung sind in kriegszerstörten Gesellschaften angebracht

27 Siehe Volker Rittberger, 2009, a.a.O., S. 47-54 (hier S. 51).

28 Siehe dazu grundlegend z.B. Klaus Schlichte: Der Staat in der Weltgesellschaft. Politische Herrschaft in Afrika, Asien und Lateinamerika. Frankfurt am Main/ New York: Campus 2005.

29 Siehe z.B. Tobias Debiel/Daniel Lambach: Global Governance as Selfdeception: How the Western State-building Project Neglects Local Politics, und Volker Böge: Challenging the Weberian Concept of the State: Lessons Learned from a Far-away South Sea Island, beide in Michael Brzoska/Axel Krohn (Hg.): Overcoming Armed Violence in a Complex World, Opladen: Budrich University Press 2009, S. 163-184 bzw. 205-223. Bei der Tagung der International Peace Research Association (IPRA) vom 6.-10.7.2010 in Sydney widmete sich die "Development and Peace Commission" schwerpunktmäßig diesem Thema. Die dort vorgestellten Überblicksanalysen und Fallstudien werden in einer Buchpublikation erscheinen. 
und erfolgversprechend? Wie betten sich diese in die jeweiligen gesellschaftlichen und kulturellen Traditionen ein? Wer sind die lokalen Träger solcher Prozesse und wie können sie von externen Akteuren unterstützt werden? Welche Rolle kommt zivilen Ansätzen zu und welche Unterstützungsfunktion können militärische Akteure bei der Friedenskonsolidierung übernehmen? Welche organisatorischen Voraussetzungen und Ausbildungen sind dafür erforderlich?

Um Antworten auf diese Fragen zu finden, muss die Interaktion von internen und externen Akteuren in den Blick genommen werden. Ein anderes, häufig zu wenig beachtetes Feld sind die grenzüberschreitenden Verflechtungen der Bemühungen um Friedenskonsolidierung.

Zahlreiche Diskussionen - internationale und nationale - zwischen Friedensforschung und Praxis haben auf mangelnde Kohärenz, Effektivität und konzeptionelle Probleme bei internationalen Friedensmissionen hingewiesen. Auch der Sinn und Zweck von internationalen Protektoraten wurde kritisch reflektiert, angesichts der Erkenntnis, dass Missionen in manchen Nachkriegsregionen Strukturen errichtet haben, die den Aufbau lokaler Fähigkeiten eher behinderten als beförderten. Zudem wurde deutlich, dass sich die Forschung bisher noch zu wenig mit der Bedeutung asymmetrischer Strukturen und Herrschaftsverhältnisse für die Konflikttransformation auseinandergesetzt hat.

Über die Bewertung von Maßnahmen der Friedenskonsolidierung muss also neu nachgedacht werden. Dazu gehört es zum einen, nachvollziehbare Kriterien für Erfolge und Misserfolge zu entwickeln. Zum anderen müssen aber auch die ideologischen Prämissen, die den Interventionen zugrunde liegen, kritisch hinterfragt werden. Die Maßstäbe wurden in der Vergangenheit häufig ad hoc entwickelt oder waren eng an die Voraussetzungen des liberalen Modells gekoppelt. Wenig Beachtung fanden dabei die bereits erwähnten Verdrängungseffekte auf andere Gewaltformen. Die Entstehung derartiger Effekte, aber auch die Frage, wie sie verhindert werden können, ist ein wichtiges Feld für weitere Forschung. Dieses ist eng verknüpft mit der Frage nach dem Umgang mit bzw. der Prävention von Terrorismus. Gleichzeitig sollten Befunde aus der Kriegsursachenforschung ernst genommen und weiter verfolgt werden, welche die neue Dynamik im internationalen Kriegsgeschehen keineswegs nur auf terroristische Strukturen, sondern auch auf den „war against terror“, der ebenfalls mit Waffengewalt (u.a. in Afghanistan sowie in den pakistanischen Grenzgebieten) ausgetragen wird, zurückführen. ${ }^{30}$

Letztlich geht es auch darum, die Politik davor zu warnen, Blaupausen anzuwenden oder schematische Modelle in ungeeignete Kontexte zu transferieren. Die Friedensforschung sollte sie mit ihren Ergebnissen darin unterstützen, passfähigere Strategien der Friedenskonsolidierung zu entwerfen, die den jeweiligen lokalen Gegebenheiten Rechnung tragen. Dabei ist vor allem weitere Expertise erforderlich, um zu bewerten, in welcher Abfolge Maßnahmen zum Wiederaufbau von Institutionen der Verwaltung und Rechtspflege, zur Herstellung von Sicherheit, zur wirtschaftlichen Entwicklung, Partizipation

$30 \mathrm{Vgl}$. Tara Cooper et al (forthcoming), a.a.O. und Demokratisierung bis hin zu Dialogstrukturen und Aussöhnungsprozessen zwischen den Konfliktparteien umgesetzt werden sollten. Aber auch die Stärken und Schwächen der an Maßnahmen der Friedenskonsolidierung beteiligten Akteure (dazu gehören sowohl internationale Organisationen als auch Regierungen und zivilgesellschaftliche Initiativen) müssten noch genauer analysiert werden.

\section{Transitional Justice und Aussöhnung}

Auch im Hinblick auf die Möglichkeiten der Aussöhnung innerhalb und zwischen kriegszerstörten Gemeinwesen ergeben sich zahlreiche Forschungsdesiderata. Damit verknüpft sich auch die Frage, wie Maßnahmen der „transitional justice“ (TJ) (Mechanismen der Strafverfolgung von Kriegs- und Menschenrechtsverletzungen, der Wahrheitsfindung und der Aufarbeitung von Vergangenheit) Nachkriegsgesellschaften beeinflussen und welche Bedingungen gegeben sein müssen, damit sie Friedenskonsolidierung unterstützen können.

Seit Anfang der 1990er Jahre haben sich Forschung und Praxis verstärkt mit der Frage befasst, wie Gesellschaften, die Krieg oder Gewaltherrschaft erfahren haben, ihre Vergangenheit aufarbeiten und wie sie langfristig zu Prozessen der Aussöhnung gelangen können. Konzepte aus dem Umfeld der „transitional justice", die ursprünglich in der Menschenrechtspraxis und -forschung entwickelt wurden, prägen inzwischen akademische Debatten über Demokratisierung und Staatsbildung und haben auch in die Friedensforschung Eingang gefunden. ${ }^{31}$ Strafverfolgung und juristische Untersuchung von Kriegsverbrechen, Faktenerhebung, Wahrheitskommissionen, Überprüfung von Entscheidungsträgern und Verwaltungen, Restitution von Eigentum und Reparationen sowie Maßnahmen zur Förderung historischer Aufarbeitung in den Bereichen Kultur und Bildung wurden überdies inzwischen auch zu zentralen Bestandteilen der Agenden und Reformbemühungen von internationalen Organisationen, Geldgebern und Beratungsagenturen für Gesellschaften, die sich im Übergang vom Krieg zum Frieden oder von autoritären zu demokratischen Regimen befinden.

Auch das Konzept der Versöhnung oder Aussöhnung (reconciliation) hat einen erheblichen Bedeutungszuwachs erfahren. Es wird häufig davon ausgegangen, dass für dauerhaften Frieden neben Abkommen, die auf der Regierungsebene (top-down) vereinbart werden, gesellschaftliche Prozesse des Dialogs und der Vertrauensbildung sowie der Aufbau von Beziehungen und Prozesse der Heilung erforderlich sind. Das Ziel ist dabei, ungelöste Elemente von Konflikten zu bearbeiten und damit zu verhindern, dass ein Friedensabkommen wieder in Frage gestellt wird und erneut Gewalt ausbricht. Die DSF hat in der Vergangenheit bereits einige Fallstudien und vergleichende

31 Vorreiter waren hier das United States Institute of Peace, das International Center for Transitional Justice sowie das South African Centre for the Study of Violence and Reconciliation. Auch Swisspeace (Bern) und das Center for Justice and Reconciliation in Den Haag haben hierzu geforscht und publiziert. Das Peace Research Institute in Oslo und die University of Wisconsin-Madison haben Datenarchive zum Thema erstellt. 
Forschungsvorhaben zu diesen Themen gefördert. ${ }^{32}$ Sie werden auch in Zukunft weiterhin Relevanz behalten.

Die Diskurse über „transitional justice“ und Aussöhnung überlappen sich, sind jedoch keineswegs deckungsgleich. Sie bieten Potenziale für die Erforschung zahlreicher, die Friedensforschung interessierender Fragen zur Friedenskonsolidierung und Gewaltprävention. Gleichzeitig müssen sie auch kritisch hinterfragt und methodisch weiter entwickelt werden. Eine Herausforderung besteht darin, die Erforschung von Prozessen der Aussöhnung von zielgebundenen Annahmen und moralischen Aspekten (wie etwa dem christlichen Prinzip der Vergebung) zu befreien, von der individualpsychologischen auf die gesellschaftliche Ebene zu beziehen und stärker politik- und sozialwissenschaftlich zu fundieren. Vor allem der Untersuchung der Zusammenhänge zwischen Konflikt und kultureller Identität sowie des Umgangs mit unterschiedlichen historischen Narrativen kommt entscheidende Bedeutung zu. In der Praxis hat sich gezeigt, dass Dialogprojekte und der Austausch unterschiedlicher Narrative Aussöhnung befördern können. Gleichzeitig wurde deutlich, dass solche Initiativen in gespaltenen Gemeinwesen unter Umständen auch kontraproduktiv wirken können. Bei der Bewertung der Bedingungen und Zeitpunkte, in denen derartige Ansätze friedensfördernd wirken, können sowohl die Friedenspädagogik als auch sozialpsychologische Forschungsansätze einen wichtigen Beitrag leisten.

Zudem muss verstärkt danach gefragt werden, wie sich unterschiedliche „Transitional justice“-Mechanismen auf die jeweiligen Gesellschaften auswirken. Zwar wurden sie in den vergangenen Jahren umfangreich im Hinblick auf ihre völkerrechtlichen und rechtlich-normativen Aspekte hin erforscht. Aber die Auswirkungen auf die Menschen und sozialen Bezüge, auf die sie sich beziehen, sind bislang kaum systematisch betrachtet worden. Oft wird Tribunalen und Wahrheitskommissionen eine pauschale friedensfördernde Wirkung unterstellt, die allerdings meist empirisch kaum nachgewiesen werden kann. ${ }^{33}$ Es geht hier also um eine belastbare Bewertung von Erfolgen und Misserfolgen.

In den Themenfeldern „transitional justice“ und Aussöhnung wird es daher in der Zukunft vor allem darum gehen, die empirische Basis durch systematische Langzeitanalysen und länderübergreifende Vergleichsstudien zu verbreitern, welche die gesellschaftlichen Wirkungen und die Friedens- und Konfliktverträglichkeit der vorgeschlagenen Instrumente näher in den Blick nehmen. Dabei ist es auch wichtig, die Interaktion der unterschiedlichen an solchen Prozessen beteiligten internationalen, gesellschaftlichen und politischen Akteure zu ana-

32 Siehe etwa Susanne Buckley-Zistel. Between Past and Future. An Assessment of the Transition from Conflict to Peace in Post-genocide Rwanda. Osnabrück 2008 (Forschung DSF Nr. 15).

33 Einige Überblicksstudien haben kürzlich offengelegt, wie dünn - trotz zahlreicher vorliegender Fallstudien - die empirische Datenbasis bislang ausfällt: David Backer: Cross-National Comparative Analysis, in: Hugo van der Merwe/Victoria Baxter/Audrey R. Chapman (Hg.): Assessing the Impact of Transitional Justice. Challenges for Empirical Research. Washington DC: US Institute of Peace 2009, S. 23-89; Oskar N.T. Thoms/James Ron/Roland Paris: The Effects of Transitional Justice Mechanisms. A Summary of Empirical Research Findings and Implications for Analysts and Practitioners. Working Paper. Ottawa: Centre for International Policy Studies, University of Ottawa 2008. lysieren, um deren Potenzial und Reichweite zu bestimmen. ${ }^{34}$ Auch diesen wissenschaftlichen Herausforderungen muss sich die Friedensforschung in der Zukunft stellen.

\section{Untersuchung der Transformation von Gewalt- akteuren}

Neuere Forschungen haben gezeigt, dass das globale Konfliktgeschehen nicht mehr in erster Linie von staatlichen Akteuren (Armeen und paramilitärischen Einheiten, die auf Weisungen von Regierungen agieren), sondern von einer Zunahme nichtstaatlicher Gewaltakteure beherrscht wird (dazu gehören u.a. bewaffnete Rebellengruppen, Milizen, die lokal oder regional einflussreichen Warlords dienen, sowie terroristische Gruppierungen oder mafiöse Netzwerke). Mit dem Zerfall staatlicher Strukturen erhalten nichtstaatliche Gewaltakteure zudem vielerorts gesellschaftliche Legitimation, indem sie Schutzfunktionen übernehmen und Orientierung bieten. Das Thema erweist sich zunehmend auch für die Praxis der Entwicklungspolitik als relevant. ${ }^{35}$

Bislang wird die Frage, ob und in welcher Form solche Akteure in Friedensverhandlungen einbezogen werden können, in der Forschung und in der Politik kontrovers diskutiert. ${ }^{36}$ Das gilt erst recht für die Frage, inwieweit diese Akteure reformierbar sind, beziehungsweise unter welchen Bedingungen sie sich zu politischen Akteuren transformieren, die sich an politische Regeln des Konfliktaustrags halten, und wie sie in (wieder) aufzubauende Gemeinwesen integriert werden können. Neben vergleichenden Untersuchungen sind hier auch mehr praxisorientierte Zugänge und Ansätze der Aktionsforschung gefragt. ${ }^{37}$

Die Frage, auf welcher Grundlage sich Individuen und Gruppen in Bürgerkriegen für einen Gewaltverzicht entscheiden und welche Erfahrungen sich daraus für die Praxis der Drittparteienintervention ableiten lassen, ist schließlich für internationale Organisationen sowie informelle Netzwerke und Verhandlungsführer, die sich um Kriegsbeendigung bemühen, von zentraler Bedeutung. Die Einbindung von Gewaltakteuren in politische Verhandlungsprozesse gilt als Schlüssel für die Beilegung von bewaffneten Konflikten. Außerdem ist davon auszugehen, dass die Chancen für eine langfristige Friedenskonsolidierung steigen, wenn es gelingt, Gewaltakteure zu transformieren und dauerhaft zu integrieren. Jedoch ergibt sich darauf haben vor allem Menschenrechtsinitiativen hingewiesen - bei ihrer Einbindung auch eine Reihe von Zielkonflikten. Diese sollten in der weiteren Forschung zu den Möglichkeiten

34 Vgl. dazu David Backer: Civil Society and Transitional Justice. Possibilities, Patterns and Prospects, in: Journal of Human Rights 3/2003, S. 297-313.

35 Vgl. Jörn Grävingholt/Claudia Hofmann/Stefan Klingebiel: Entwicklungspolitik im Umgang mit nichtstaatlichen Gewaltakteuren. Studies 24, Bonn Deutsches Institut für Entwicklungspolitik 2007.

36 Im Friedensgutachten 2010 wird dies im Abschnitt „Vom Bürgerkrieg zur politischen Konkurrenz?" anhand von zahlreichen Fallbeispielen diskutiert, vgl. Christiane Fröhlich et al. (Hg.): Friedensgutachten 2010. Berlin/Münster: LIT-Verlag 2010, S. 119-286.

37 Vgl. Veronique Dudouet: Mediating Peace with Proscribed Armed Groups. Washington DC: United States Institute for Peace (Special Report No. 239) 2010. http://www.usip.org/resources/mediating-peace-proscribed-armedgroups. 
und Grenzen der Beteiligung bewaffneter Akteure ebenfalls Berücksichtigung finden.

\section{Institutionen und Instrumente der Gewaltprä- vention}

Institutionelle Aspekte der Gewaltprävention hatten in der bisherigen Forschungsförderung der DSF zu Recht ein besonderes Gewicht. Untersuchungsgegenstände waren beispielsweise die Vereinten Nationen und die Europäische Union aber auch funktionale Institutionen wie das Nicht-Weiterverbreitungsregime für Nuklearwaffen. Dabei bestätigte sich, dass institutionelle Arrangements wichtige Voraussetzungen für erfolgreiche Gewaltprävention wie auch für Konfliktmanagement und Friedenskonsolidierung darstellen. Gefördert wurden auch Projekte, die sich mit den Instrumenten beschäftigten, die Akteuren in diesem Umfeld zur Verfügung stehen, zum Beispiel Friedenseinsätze zur Absicherung von Friedensabkommen.

Dem Thema Gewaltprävention wird jedoch insgesamt sowohl von der Politik als auch von der Wissenschaft bislang immer noch zu wenig Bedeutung beigemessen. Deutschland hat mit seinem 2004 veröffentlichten „Aktionsplan zivile Krisenprävention, Konfliktlösung und Friedenskonsolidierung“ sowohl hierzulande als auch international viel beachtete Impulse gegeben. Allerdings ist der Aktionsplan auch, nicht zuletzt aus den Reihen der Friedensforschung, als unzureichend kritisiert worden. So wurde die fehlende konzeptionelle Einbindung in ein größeres Gesamtkonzept deutscher Friedens- und Sicherheitspolitik beklagt und eine stärkere Bündelung der verschiedenen Aktivitäten von Bundesministerien und nachgeordneten Organisationen angemahnt. ${ }^{38}$ Zudem wurden unabhängige Evaluierungen vorgeschlagen, um eine Bewertung von Erfolgen und Misserfolgen zu ermöglichen. Vor allem wurde eine effektivere und offensivere Öffentlichkeitsarbeit gefordert, die sich darauf konzentriert, konstruktive Ansätze der Prävention und Friedenskonsolidierung sichtbarer zu machen. ${ }^{39}$

Im Einklang mit dem Auftrag der DSF ist es für die Forschung dringend geboten, Institutionen und Instrumente der Gewaltprävention eingehender zu untersuchen, um diese weiter $\mathrm{zu}$ entwickeln. Auch in Zukunft ist die Friedensforschung aufgefordert, in Kooperation mit staatlichen und nichtstaatlichen Akteuren, Handlungskonzepte zu überprüfen und friedenspolitische Alternativen zu entwickeln. Das gilt sowohl für Deutschland als auch für institutionelle Arrangements auf der Ebene von Regionalorganisationen wie der Europäischen Union und der Organisation für Sicherheit und Zusammenarbeit in Europa (OSZE) sowie für die Vereinten Nationen. Auch über neue und alternative Institutionen und funktionale Regime lohnt

38 Siehe etwa Peter Croll/Tobias Debiel/Stefan Klingebiel: Plädoyer für eine integrative Sicherheitsstrategie. Warum die Bundesregierung sich im europäischen Kontext mit zivilen Akzenten in der Sicherheitsstrategie stärker profilieren sollte. Bonn/Duisburg, 2007, und Frank A. Stengel/Christoph Weller: Action Plan or Faction Plan? Germany's Eclectic Approach to Conflict Resolution. In: International Peacekeeping 1/2010, S. 93-107.

39 Siehe das Memorandum zur Bundestagswahl 2009, das von den im zivilgesellschaftlichen Beirat vertretenen Friedensforschern veröffentlicht wurde. http://www.bicc.de/press/press-2009/presseerklaerung-memorandum-bundestagswahl-2009.html. das Nachdenken mit den Instrumenten wissenschaftlicher Forschung.

Seit den 1990er Jahren ist eine Reihe von Instrumenten für Gewaltprävention neu entwickelt oder ausgebaut worden..$^{40}$ Die internationale Strafgerichtsbarkeit, die internationale Schutzverantwortung („resonsibility to protect“41) oder auch zielgerichtete Sanktionen und Frühwarnsysteme wurden entwickelt. Diese Instrumente sind teilweise bereits auch in DSF-geförderten Forschungsvorhaben untersucht worden. Aber noch ist die empirische Grundlage in diesen Bereichen dünn und sollte durch weitergehende und methodisch fundierte Untersuchungen angereichert werden. Weiterhin besteht eine Herausforderung für die Forschung auch darin, sich eingehender mit Beispielen gelungener Prävention und Friedenskonsolidierung zu befassen. Dazu gehört auch die Analyse von Fällen, in denen gewaltfreie Aktionen zu gesellschaftlichem Wandel und Konfliktbeilegung beigetragen haben.

\section{Grundlagen dauerhaften Friedens}

Friedensforschung ist, wie eingangs betont, nicht nur Kriegsoder Gewaltverhütungswissenschaft, sondern sie beschäftigt sich auch mit den Grundlagen für friedliches Zusammenleben. Auch die DSF hat sich dies zur Aufgabe gemacht. Wichtig ist dabei die Feststellung, dass menschliche und soziale Interaktion, wie auch die Beziehungen zwischen Staaten, nicht ohne Konflikte zu denken sind, und dass Konflikte vielfach sogar einen wichtigen Bestandteil von sozialer Entwicklung und Fortschritt bilden. Gleichzeitig ist es erforderlich, sie in Bahnen zu lenken, in denen sie gewaltfrei ausgetragen werden. ${ }^{42}$ Konflikte sind demnach allgegenwärtig, und doch zeigt sich, dass tatsächlich nur verhältnismäßig wenige davon unter Anwendung oder Androhung von Gewalt ausgetragen werden: „Ein großer, wenn nicht der größte Teil von menschlichen Lebenschancen wird hingegen gewaltlos zugeteilt. “43 Krieg und Gewaltkonflikte sind also nach dem Verständnis der Friedensforschung nicht naturgegeben, sondern Ausdruck des Versagens von Politik. Diese steht in der Verantwortung, Krieg zu verhüten und internationale Beziehungen so zu gestalten, dass auf diese Form der Auseinandersetzung verzichtet wird.

Frieden wurde von der Forschung als Prozess definiert, der von einer Abnahme von Gewalt (nicht nur bewaffneter, sondern

40 Siehe etwa verschiedene Beiträge in Andreas Fischer-Lescano/Hans-Peter Gasser/Thilo Marauhn/Natalino Ronzitti (Hg.): Frieden in Freiheit. Festschrift für Michael Bothe zum 70. Geburtstag. Baden-Baden: Nomos 2009.

41 Nachdem der frühere UN-Generalsekretär Kofi Annan die Frage aufgeworfen hatte, wie mit dem Prinzip der Souveränität angesichts massiver Menschenrechtsverletzungen umzugehen sei, wurde die International Commission on Intervention and State Sovereignty (ICSS) eingerichtet, die unter dem Vorsitz des ehemaligen australischen Außenministers Evans und des UN-Sondergesandten Sahnoun den Bericht „Responsibility to Protect“ vorlegte (vgl. http: www.iciss.ca/pdf/commission-Report.pdf). Die UN-Mitgliedstaaten kamen 2005 überein, dass eine Schutzverantwortung für die Bevölkerungen vor Völkermord, Kriegsverbrechen oder ethnischer Säuberung gegeben ist und dass die Vereinten Nationen eingreifen müssen, wenn Staaten dieser Verantwortung nicht nachkommen.

42 Vgl. zu diesen Überlegungen Ernst-Otto Czempiel: Internationale Politik. Ein Konfliktmodell. Paderborn, 1981; Dieter S. Lutz: Der Friede als Ernstfall oder das Recht des Stärkeren, in: Ders. (Hg.): Die Stärke des Rechts gegen das Recht des Stärkeren. Baden-Baden: Nomos, 2003.

43 Volker Rittberger 2009, a.a.O, S. 47-54 (hier S. 48) 
auch struktureller Gewalt) ${ }^{44}$ und einer zunehmenden Verrechtlichung der internationalen Beziehungen gekennzeichnet ist. Die Bedingungen für die Zivilisierung ${ }^{45}$ sozialer und zwischenstattlicher Beziehungen wurden untersucht und definiert, aber auch kontrovers diskutiert. Gleichzeitig wurde vielfach auf die Notwendigkeit zunehmender Verteilungsgerechtigkeit als Grundlage dauerhaften Friedens hingewiesen, was einen ökologisch verträglichen, am Prinzip der Nachhaltigkeit orientierten Umgang mit natürlichen Ressourcen einschließt.

In den vergangenen beiden Jahrzehnten ist zudem verstärkt darauf hingewiesen worden, dass die Bedeutung des Geschlechterverhältnisses und die Art und Weise, wie Geschlechterrollen gesellschaftlich konstruiert und festgelegt werden, in die Untersuchung von Gewaltdynamiken und Friedensprozessen einbezogen werden müssen. Hier geht es zum einen darum zu untersuchen, wie Frauen als Akteurinnen in Friedensprozessen wahrgenommen und gestärkt werden können, zum anderen um die unterschiedlichen Rollenkonstruktionen, mit denen Männlichkeit und Weiblichkeit sozial und kulturell belegt werden. Diese sind in ihrer Bedeutung für Gewaltkonflikte bzw. die Gestaltung von Alternativen zu analysieren. In der Praxis der vergangenen Jahre hat sich gezeigt, dass Forschungs- und Politikansätze, die Gender-Dimensionen aussparen, riskieren, verfehlte Strategien für Friedensprozesse zu entwerfen. Zahlreiche Defizite internationaler Friedensmissionen sind hierzu aus der Praxis belegt. ${ }^{46}$ Zur Bedeutung der Kategorie „gender“ bei der Untersuchung von Gewaltdynamiken und Friedensprozessen wurde vor allem in US-amerikanischen und skandinavischen Forschungszusammenhängen umfangreiche Expertise erstellt. Auch deutsche Forschungseinrichtungen haben dazu inzwischen einschlägig publiziert. ${ }^{47}$ Insgesamt wäre aber zu wünschen, dass gender-bezogene Untersuchungsansätze noch stärker als Querschnittsaufgabe begriffen und nicht nur vom weiblichen Teil der Forschungswelt aufgegriffen werden.

Alle bis hierher genannten Fragen haben zentrale Bedeutung für das Wissen über die Bedingungen von Krieg und Frieden. Die DSF wird insbesondere dann gefordert sein, wenn innovative Ideen und Forschungsergebnisse zu relevanten Grundfra-

44 Vgl. Johan Galtung: Violence, Peace and Peace Research, in: Journal of Peace Research 3/1969, S. 167-191, und ders.: Strukturelle Gewalt: Beiträge zur Friedens- und Konfliktforschung. Reinbek bei Hamburg: Rowohlt 1975.

45 Dieter Senghaas entwickelte das „zivilisatorische Hexagon“ zur Beschreibung der Bausteine für eine friedliche Gesellschaft, bestehend aus: (1) dem Gewaltmonopol als Folge der Entprivatisierung von Gewalt, (2) Rechtsstaatlichkeit, (3) Demokratischer Partizipation, (4) Sozialer Gerechtigkeit einschließlich der Sicherung der sozialen Menschenrechte, (5) Konstruktive Konfliktkultur, (6) Interdependenzen und Affektkontrolle. Vgl. Dieter Senghaas: The Civilisation of Conflict: Constructive Pacifism as a Guiding Notion for Conflict transformation. In: Alex Austin/Martina Fischer/Norbert Ropers (Hg.): Transforming Ethnopolitical Conflict. The Berghof Handbook, Wiesbaden: VS-Verlag 2004 S. $25-40$.

46 Siehe Martina Fischer: Gender, Gerechtigkeit und Sicherheit in Nachkriegsgesellschaften, in: Sicherheit und Frieden (S+F) 1/2010, S. 21-28.

47 Siehe Sarah Clasen/Susanne Zwingel: Geschlechterverhältnisse und Gewalteskalation, in: PVS Sonderheft Identität, Institution und Ökonomie, Ursachen innenpolitischer Gewalt, Wiesbaden: VS Verlag 2009, S. 128-149; Christine Eifler/Ruth Seifert (Hg.): Gender Dynamics. Post-Conflict Reconstruction. Frankfurt a.M.: Peter Lang 2009; Cilja Harders: Geschlecht und Gewaltminderung: Konfliktbearbeitung durch Veränderung von Machtverhältnissen, in: Astrid Sahm/Egbert Jahn/Sabine Fischer (Hg.): Die Zukunft des Friedens weiterdenken - Perspektiven der Friedens- und Konfliktforschung. Wiesbaden: VS Verlag 2005, S. 495-518; Ann Judith Tickner: Gendering World Politics. New York: Columbia University Press 2001. Einen Überblick über den Zusammenhang von Gender, Sicherheit und Friedenskonsolidierung gibt das Themenhaft Gender \& Sicherheit der Zeitschrift Sicherheit und Frieden $1 / 2010$. gen erarbeitet werden, die einen starken Bezug zum Kernthema der Friedensforschung, der Gewaltverhinderung und Gewaltminderung, haben. Auch in dieser Grundlagenforschung bietet sich die Zusammenarbeit von Friedensforscherinnen und -forschern mit Wissenschaftlern aus anderen Disziplinen an.

\section{Weitere Zukunftsthemen}

\section{Ressourcenkonflikte}

In den vergangenen Jahren widmete sich die Friedensforschung bei der Untersuchung von Gewaltkonflikten zunehmend auch der Bedeutung des Wettstreits um Ressourcen. ${ }^{48}$ Die Bedeutung des Themas wird zunehmen, denn mit dem Rückgang der Reserven an Öl und Gas werden weitere zukünftige Gewaltkonflikte zwischen Staaten prognostiziert. ${ }^{49}$ Auch in der Folge des Klimawandels werden häufig Kriege um natürliche Ressourcen, insbesondere um Wasser, erwartet.

Viele dieser Voraussagen sind allerdings alarmistisch und stehen wissenschaftlich auf wackeligen Füßen. Gerade dadurch ergibt sich aber ein erheblicher Bedarf für kritische Forschung. Sie sollte Thesen, die aus der Ressourcenverknappung automatisch eine Zunahme des Kriegsgeschehens folgern, zurückweisen, ohne dabei jedoch die Bedeutung von Ressourcen für verschiedene Wirtschaftszweige in modernen Gesellschaften zu verkennen.

Darüber hinaus sollte aber vor allem das Kooperationspotenzial in diesem Feld zu einem zentralen Forschungsgegenstand gemacht werden. Am Beispiel grenzüberschreitender Gewässer zeigt sich, dass Konflikte um Nutzung und Zugänge auch unter Wahrung der Interessen aller Beteiligten geregelt werden können. ${ }^{50}$ Aber auch bezogen auf andere Ressourcen und für die globale Ressourcenwirtschaft insgesamt sind inzwischen Modelle kooperativer, nachhaltiger Nutzung entworfen worden. ${ }^{51}$

Die Befunde zu Bürgerkriegen und die Prognosen zukünftiger Ressourcenkonflikte im Zuge des Klimawandels deuten darauf hin, dass Frieden ohne eine global gerechte und nachhaltige Ressourcennutzung langfristig kaum zu haben sein wird. Die Erforschung der Grundlagen und Bedingungen kooperativer Umgangsformen wird damit zu einem wichtigen Themenfeld für die Friedensforschung.

\section{Klimawandel}

Den Klimawandel als Zukunftsthema zu bezeichnen mag überraschen - sind doch erste Auswirkungen wie globaler

48 Siehe als Überblick etwa Michael Ross: What Do We Know About Natural Resources and Civil War?, in: Journal of Peace Research 3/2004, S. 337-356.

49 Siehe etwa Michael Klare: Resource Wars: The New Landscape of Global Conflict. New York: Owl Books 2002.

50 Siehe z.B. Alexander Carius/Geoffrey Dabelko/Aaron Wolf: Water, Conflict, and Cooperation. Environmental Change and Security Project Report. No.10, 2004 , S. 60-66.

51 Siehe Raimund Bleischwitz: Präventive Ressourcensicherheitspolitik: Initiativen und Anforderungen an ein Global Governance-System, in: Sicherheit und Frieden (S+F) 4/2009, S. 231-237. 
Temperaturanstieg bereits in der Gegenwart feststellbar. Aber diese Auswirkungen sind marginal im Vergleich zu dem, was Klimaforscher bis zum Ende dieses Jahrhunderts erwarten, selbst wenn es gelingen sollte, Gegenmaßnahmen rasch und umfassend einzuleiten. Aktuell stehen wir erst am Anfang der Forschung zu den Auswirkungen. Es liegen zwar gesicherte Erkenntnisse vor über die unmittelbaren geophysikalischen Phänomene wie Temperaturentwicklung, Regenverteilung und Extremwetterereignisse, aber darüber hinaus wissen wir wenig. Erste tentative Einschätzungen der Veränderungen in der Vegetation oder den Häufigkeiten von Krankheiten in bestimmten Regionen werden präsentiert. Aber eine umfassende Analyse der vielfältigen Auswirkungen des Klimawandels auf die Umwelt und die menschlichen Lebensbedingungen kann in absehbarer Zeit nicht erstellt werden, dazu ist der Klimawandel ein zu ungewöhnlicher Vorgang.

Noch schwieriger sind Vorhersagen über Änderungen im individuellen und kollektiven menschlichen Verhalten. Die Analyse vergangener Umweltveränderungen ist dafür nur beschränkt hilfreich, da sie auch in den wenigen Fällen großer Katastrophen kaum das Ausmaß erreicht haben, das für den Klimawandel erwartet wird. ${ }^{52}$ Dasselbe gilt für Vorhersagen auf der Grundlage gesellschaftlicher Stresssituationen. Analysen historischer Konstellationen von Umweltfaktoren und Konflikten sind also nur bedingt tauglich für die Prognose zukünftiger Entwicklungen, zumal sich die gesellschaftlichen und technischen Bedingungen zu Beginn des 21. Jahrhunderts in so rasanter Weise verändern, dass es in der Menschheitsgeschichte dafür keinen Vergleich gibt. ${ }^{53}$

Trotz der methodischen Probleme und Herausforderungen ist auch die Friedensforschung aufgefordert, sich noch stärker als bisher mit den Folgen des Klimawandels für Gewaltprävention und Friedensbildung zu befassen. Das wird nur in Zusammenarbeit mit der Klimaforschung möglich sein. Die Friedensforschung wird sich neuen Methoden (z.B. der Szenarioanalyse) öffnen und diese gleichzeitig kritisch hinterfragen müssen.

Umgekehrt kann die Friedensforschung selbst auch wertvolle Hinweise dafür liefern, wie Gewalt und Krieg - häufig als Folgen des Klimawandels prognostiziert - vermieden werden können. Die Erkenntnisse der Friedensforschung zu Gewaltprävention und Friedensförderung, zu Institutionen und Instrumenten werden in Zeiten des beschleunigten Wandels der natürlichen und gesellschaftlichen Umwelt an Bedeutung gewinnen.

\section{Veränderungen der globalen Machtarchitektur}

Nach einigen voreiligen Ankündigungen in den letzten Jahrzehnten dürfte die Prognose dieses Mal zutreffen: Wir erleben Zeiten weltpolitischer Neuordnung. Die wirtschaftliche und politische Bedeutung Chinas, Indiens und mit Abstrichen auch

52 Halvard Buhaug/Ole Magnus Theisen/Nils Petter Gleditsch: 'Implications of Climate Change for Armed Conflict', paper prepared for the Social Dimensions of Climate Change Program. Washington, DC: World Bank, Social Development Department 2008. http://siteresources.worldbank.org/INTRANETSOCIALDEVELOPMENT/Resources/SDCCWorkingPaper_Conflict.pdf.

53 Siehe etwa Larry Diamond: Collapse. How Societies Choose to Fail or Succeed. New York: Viking 2005, und Harald Welzer: Klimakriege. Wofür im 21. Jahrhundert getötet wird. Frankfurt a.M.: Fischer Verlag 2007.
Brasiliens wächst. Die Vereinigten Staaten und Europa werden nicht auf Dauer internationale Organisationen dominieren können, so wie es seit dem Ende des Kalten Kriegs der Fall war.

Was aber folgt aus der globalen Neuordnung für Fragen der Gewaltprävention und der Friedenskonsolidierung? Werden die vor allem im Westen entwickelten Normen zu Krieg und Frieden, Gewalt und Ordnung in Frage gestellt werden? Wird es zu neuen Konfliktformationen kommen oder zu einer neuen, gerechteren und friedlicheren Weltordnung? ${ }^{54}$

Diese und andere Fragen beschäftigen bereits zahlreiche Wissenschaftlerinnen und Wissenschaftler, die sich mit internationalen Wirtschaftsfragen und mit den Auswirkungen großer Machtverschiebungen befassen. Dieses Thema wird vor allem für Sozial- und Wirtschaftswissenschaftler an Bedeutung gewinnen. Aber auch Geschichtswissenschaftler werden wieder vermehrt über historische Parallelen schreiben. Der Friedensforschung wächst die Aufgabe zu, in Zusammenarbeit mit anderen Wissenschaften die wahrscheinlichen Auswirkungen der Veränderungen im Machtgefüge zu analysieren und ihre Bedeutung für Gewaltprävention und Friedensförderung zu untersuchen.

Einige wichtige Themen sind mit der weiteren Entwicklung internationaler Normen und dem faktischen und/oder formellen Umbau von relevanten Institutionen wie den Vereinten Nationen bereits benannt. Aber es ist davon auszugehen, dass das gesamte Themenspektrum der Friedensforschung, von der Abrüstung über militärische Interventionen bis hin zur Friedenspädagogik davon berührt sein wird.

\section{Schlussbetrachtung}

Die Welt ist in den letzten zehn Jahren - dank weltweiter Anstrengungen zur Gewaltvorbeugung und Kriegsverhütung zumindest vorübergehend ein wenig friedlicher geworden. Dazu hat auch die Friedensforschung mit ihren Erkenntnissen zu gewaltfördernden Faktoren, zur Bedeutung internationaler Institutionen oder zu den Bedingungen der Nachkriegskonsolidierung beigetragen. Wie dieser Aufriss zeigt hat, ist das Spektrum der wissenschaftlich interessanten und gesellschaftlich relevanten Themen jedoch weiterhin umfangreich und die Forschungsaufgaben, die sich daraus ergeben, sind anspruchsvoll. Im 21. Jahrhundert stehen Forschung und Politik vor zahlreichen neuen Herausforderungen. Sie müssen Vorschläge dafür erarbeiten, wie Verschiebungen in der globalen Machtarchitektur, Ressourcenkonkurrenzen und der globale Klimawandel in friedensverträglicher Weise bewältigt werden können. Dafür ist es notwendig, neue Erkenntnisse aus anderen Wissenschaften in der Friedensforschung aufzunehmen und themenzentriert weiter zu entwickeln. Darüber hinaus aber bleiben, wie dieser Beitrag auch gezeigt hat, viele „alte“ Fragen und Ansätze, für die in den letzten Jahren noch keine angemessenen und hinreichenden Antworten gefunden wurden, weil es an geeigneten

\footnotetext{
54 Siehe etwa Raphael Kaplinsky/Dirk Messner: Impact of Asian Drivers on the Developing World, in: World Development 2/2008, S. 197-209, und Daniel Flemes (Hg.): Regional Leadership in the Global System: Ideas, Interests and Strategies of Regional Powers. Aldershot: Ashgate 2010.
} 
Methoden oder Daten oder schlicht an Ressourcen für die Forschung mangelte, auch weiterhin auf der Agenda.

Die Friedensforschung verfügt über das Potenzial, wichtige Ergebnisse in den genannten Feldern zu erzielen. Dazu hat die Fördertätigkeit der DSF in den vergangenen zehn Jahren einen maßgeblichen Beitrag geleistet. Sie wird auch in Zukunft die Forschungslandschaft nach Kräften unterstützen, wobei ihr die gegenwärtig zur Verfügung stehenden finanziellen Mittel enge Grenzen setzen. Die Deutsche Stiftung Friedensforschung kann mit den bescheidenen Mitteln, die ihr aus dem vorhandenen Stiftungskapital von etwa 27 Millionen Euro zufließen, nur einzelne ausgewählte Vorhaben aus der Fülle der relevanten Themen fördern. Die bei der Gründung der Stiftung vorgesehene Erhöhung des Stiftungskapitals auf 50 Millionen Euro würde die Möglichkeiten der DSF deutlich verbessern ohne allerdings das ganze Spektrum von vielversprechenden Forschungsthemen abdecken zu können. Sie wird dauerhaft bestrebt sein müssen, gezielt Anstöße für die Behandlung drängender Forschungsfragen zu geben, wissenschaftliche Standards zu setzen, Vernetzung zu verstärken und die Verbreitung von Forschungsergebnissen zu unterstützen.

Die DSF kann nur in begrenztem Maße zur Verbreiterung der Erkenntnis in der Grundlagenforschung beitragen - zu umfangreich und vielfältig sind hier die relevanten Themen, angefangen vom Verhältnis von Gerechtigkeit zu Frieden, über die Fortentwicklung des Völkerrechts als Grundlage für eine dauerhafte internationale Friedensordnung bis hin zu Fragen des Geschlechter- und Generationenverhältnisses oder der individuellen und kollektiven Identität im Zeitalter der Globalisierung. Jedoch lassen sich im Bereich der anwendungsorientierten Forschung bisherige Schwerpunkte und Profile weiter ausbauen und neue Akzente setzen, insbesondere wenn die finanziellen Möglichkeiten der Stiftung erweitert werden. Die Forschungsleitlinien, die die DSF in den ersten zehn Jahren ihrer Existenz begleitet haben, bedürfen der Anpassung an zukunftsweisende Trends und der Ergänzung zusätzlicher Themenstellungen. Gleichzeitig wird zu klären sein, wie transdisziplinäre Forschung und interdisziplinäre Ansätze verstärkt werden können. Aber auch die Fragen, wie Nachwuchsförderung sinnvoll zu gestalten ist und wie sich der Austausch zwischen der deutschen Forschung und der internationalen Friedenswissenschaft intensivieren lässt, stehen auf der Agenda.

Die DSF wird aufbauend auf ihren bisherigen Leistungen kreative Wege finden, um die Friedensforschung in Deutschland für das 21. Jahrhundert fit zu machen. Sie wird nicht darin nachlassen und sich weiterhin beharrlich dafür engagieren, Politik und Öffentlichkeit von ihrer Vision zu überzeugen, dass kollektive Gewaltanwendung zwischen und innerhalb von Staaten langfristig obsolet und ein Leben ohne Angst und Not für alle Menschen zur Realität werden kann und muss.

\section{Kommission „Europäische Sicherheit und Zukunft der Bundeswehr" am IFSH*}

\section{EUropäische Streitkräfte}

\section{Friedensmacht Europa}

Das immer enger zusammenwachsende Europa hat sich zu einer Zone stabilen Friedens entwickelt, in der Interessenkonflikte zwischen den Mitgliedstaaten ohne die Androhung oder Anwendung militärischer Gewalt geregelt werden. Die Methode, die zu dieser Erfolgsgeschichte geführt hat, ist die Überwindung nationaler Egoismen durch Kooperation und Integration. Ihr lag die Erkenntnis zugrunde, dass angesichts der Zerstörungen durch die beiden Weltkriege die Selbstbehauptung Europas nur durch die Überwindung von Nationalismus und Krieg möglich ist. Die Befriedung Europas im Innern eröffnet die Möglichkeit, Friedenspolitik nach außen zu betreiben.

\footnotetext{
Autoren: Detlef Bald, Jörg Barandat, Michael Brzoska, Hans-Georg Ehrhart, Hans-Günter Fröhling, Hans-Joachim Gießmann, Jürgen Groß, Lars Klingbeil, Berthold Meyer, Burkhardt Müller-Sönksen, Reinhard Mutz, Winfried Nachtwei, Bernhard Rinke, Jürgen Rose, Jochen Scholz, Peter Tobiassen.
}

Die Konflikte des 21. Jahrhunderts haben einen anderen Charakter als diejenigen zu Zeiten der Ost-West-Konfrontation. Sie sind komplexer und diffuser, wirken eher indirekt und schleichend, haben aber letztlich doch einen zersetzenden Einfluss auf die internationale Ordnung. In der globalisierten Welt geht es daher nicht mehr nur um Frieden und Sicherheit innerhalb der Europäischen Union (EU), sondern auch und vor allem um Frieden und Sicherheit für, mit und durch die EU. Gefordert ist nichts weniger als ein europäischer Beitrag zur Entstehung einer kooperativen Weltordnung. Die EU steht damit vor der zentralen strategischen Gestaltungsaufgabe, in die Rolle eines internationalen Ordnungsfaktors hineinzuwachsen. Diese Aufgabe erfordert die Überwindung des nationalstaatlichen Blicks zugunsten eines integrationspolitischen Realismus, der davon ausgeht, dass angesichts der vielfältigen globalen Risiken das reflektierte Eigeninteresse der Staaten es geradezu gebietet, den aktuellen globalen Herausforderungen und Gefahren gemeinsam und auf der Grundlage des internationalen Rechts zu begegnen. 